\title{
First report of Enterobacter hormaechei with respiratory disease in calves
}

\author{
Zhicheng Wang ${ }^{\dagger}$, Lisha Duan ${ }^{\dagger}$, Fei Liu, Yun Hu, Chaoliang Leng, Yunchao Kan ${ }^{*}$, Lunguang Yao ${ }^{*}$ and Hongfei Shi ${ }^{*}$ (D)
}

\begin{abstract}
Background: Enterobacter hormaechei is commonly considered a causative pathogen for nosocomial infections and it does not usually cause diseases in animals. However, researchers have recently dissociated the pathogenic Enterobacter hormaechei from foxes and piglets. Here, the Enterobacter hormaechei was first found to be associated with respiratory disease in unweaned calves in China.

Case presentation: A 2-month-old calf was severely sick and diagnosed with respiratory infection by a rural veterinarian, and it died 5 days after treatment with penicillin $\mathrm{G}$. The lung sample was then run through histopathological analysis and pathogen isolation. The sequence analysis and biochemical tests results showed the isolated bacterium strain to be Enterobacter hormaechei, and drug sensitivity tests showed resistance to all $\beta$-lactam antimicrobials and sensitivity to quinolones. Thickened alveoli septum, inflammatory cell infiltration, and erythrocyte diapedesis around the pulmonary alveoli septum were visible in lung histopathological sections. One week later, at the same farm, another calf showed similar clinical signs, and the Enterobacter hormaechei strain was isolated from its nasal discharge; after a week of treatment with enrofloxacin, as suggested by the results of drug sensitivity tests, this calf fully recovered.
\end{abstract}

Conclusions: To the best of our knowledge, this is the first case report of calves with respiratory disease that was associated with E. hormaechei, and multi-drug resistance was observed in isolates.

Keywords: Enterobacter hormaechei, Respiratory disease, Calf, Drug resistance

\section{Background}

Enterobacter hormaechei (E. hormaechei) is a species of oxidase-negative gram-negative rods that was first identified as a unique species in 1989 [1]. E. hormaechei is widespread in many environmental niches [2]. It is commonly considered a causative pathogen of nosocomial infections [3, 4], and it does not usually cause diseases in animals other than humans. To date, only two strains associated with disease in domestic animals have been reported: one strain of $E$. hormaechei associated with uterine infection has been identified from a dead fox [5] and another strain from the excrement of piglets with diarrhea has been isolated [6]. However, there is little evidence of $E$. hormaechei with disease in calves. In this

\footnotetext{
* Correspondence: kanyunchao@163.com; lunguangyao@163.com; kcn1@163.com

†Zhicheng Wang and Lisha Duan contributed equally to this work. Henan Provincial Engineering Laboratory of Insects Bio-reactor, Henan Provincal Engineering and Technology Center of Health Products for Livestock and Poultry, China-UK-NYNU-RRes Joint Libratory of Insect Biology, Nanyang Normal University, Nanyang, People's Republic of China
}

work, we describe the first E. hormaechei clinical isolates from lung sample and nasal secretion in the calves. To our knowledge, this is the first isolation of this pathogen associated with respiratory disease in ruminants.

\section{Case presentation}

Many species of bacterium, such as Pasteurella multocida, Mannheimia haemolytica, Mycoplasma bovis, and Histophilus somni, have been identified as common pathogens involved in the bovine respiratory disease complex. The aim of this report was to present pathological, microbiological, and sequence findings in a case of naturally occurring respiratory disease with $E$. hormaechei in two calves from backyard farms in the mountainous area of southern Henan Province in central China.

One 2-month-old calf (calf A) was observed to be severely sick and developed high fever $\left(41.5 \sim 41.8^{\circ} \mathrm{C}\right)$, depression, and reduced activity in Fangcheng City in December 2018. Further rural veterinary examination of

(c) The Author(s). 2019 Open Access This article is distributed under the terms of the Creative Commons Attribution 4.0 International License (http://creativecommons.org/licenses/by/4.0/), which permits unrestricted use, distribution, and 
the diseased animal showed profuse foul-smelling nasal discharge and deep abdominal respiration. It was diagnosed with bovine respiratory disease (Additional file 1). The calf was treated with penicillin G (Zhusheyong Qingmeisuna, North China Pharmaceutical Group) with $1 \times 10^{4} \mathrm{IU}$ per $\mathrm{kg}$ of b.w. in a single dose given as two doses/day for 5 days, but it died 5 days after the initiation of treatment. Then, the partial lung sample was transported to our laboratory (nearly $100 \mathrm{~km}$ away from the farm) for histopathological analysis and pathogen isolation by the streak plate method using a nutrient agar plate $[5,6]$, and after PCR detection $[5,6]$ and sequencing analysis of ten isolates the results showed only one species of bacterium was isolated. Further, the representive strain (HN18447) was then subjected to drug sensitivity tests and biochemical tests $[5,6]$. One week later, at the same farm another 4-month-old calf (calf B) showed similar clinical signs $\left(41.3-41.5^{\circ} \mathrm{C}\right.$ ). Nasal discharge samples from this calf were also transported to our laboratory for drug sensitive testing $[5,6]$, and a sensitive antibiotic (enrofloxacin) (Zhusheyong Qingmeisuna, North China Pharmaceutical Group) with $2.5 \mathrm{mg}$ per $\mathrm{kg}$ of b.w. in a single dose given as two doses/day for 5 days was chosen for the treatment of calf B. After a week of treatment, the diseased animal recovered from its respiratory infection. Sequencing analysis of ten isolates also showed only one species of bacterium was isolated, then the representive bacterial strain (HN18449) isolated from the nasal discharge was analyzed in depth as were the strains isolated from the lungs of calf A.

Histopathologically, the alveoli septum in the lungs of calf A was significantly thickened, and a large number of inflammatory cells, mainly neutrophils, were infiltrated. Erythrocyte diapedesis was observable around the pulmonary alveoli septum (Fig. 1).

Lung and nasal discharge samples obtained from the two calves yielded colonies on $5 \%$ horse serum nutrient agar after $16 \mathrm{~h}$ of incubation at $37^{\circ} \mathrm{C}$ according to culture methods in the previous research $[5,6]$. The bacterial isolates were gram-negative and oxidase-negative, compatible with the general characteristics of the genus Enterobacter. The general primer set was used to assess the 16S rRNA gene of the all isolates [7]. Then the amplified products were recovered from the agarose gel using a gel extraction kit (Omega Bio-Tek, China), and the purified amplicons were directly sequenced in both directions using an ABI automated A373 sequencer (ABI, US). The sequence data of the 16S rRNA gene of 20 isolates from calf A and calf B indicated that all were included in the same species, and the sequences of two representive strains were deposited in GenBank under accession numbers MK774673 and MK774674. Lastly, all of the sequences were compared to the NCBI

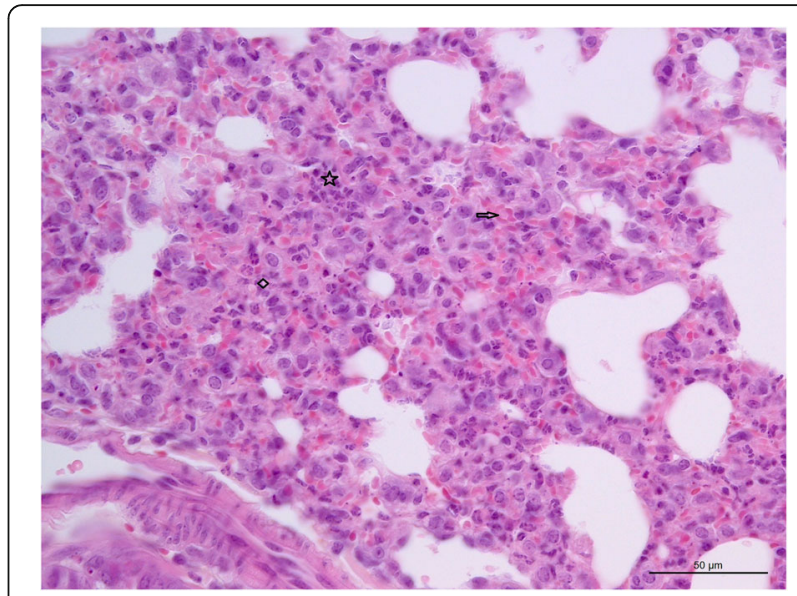

Fig. 1 Histopathological section of the lung. Alveoli septum thickening (indicated with diamond), neutrophils infiltration (indicated with asterisk) and erythrocyte diapedesis indicated with arrow of the lung from died calf. Hematoxylin and eosin stain $(\mathrm{Bar}=50 \mu \mathrm{m})$

databases using a BLAST search. The BLAST results showed that the two representive strains were both $E$. hormaechei, and the 16S rDNA sequences shared 100\% identity to the strains isolated from humans. The sequencing results of the 20 isolates indicated no other bacteria were isolated.

Susceptibility tests were performed using the previously described disk diffusion method and were interpreted in accordance with Clinical and Laboratory Standards Institute (CLSI) guidelines [8]. There were 26 antimicrobials that were tested. Briefly, the isolates' inocula were plated on Mueller-Hinton agar (Beijing Solarbio Science \& Technology Company, China), and the diameter of the inhibitive zone was determined following a 16-h incubation period at $37^{\circ} \mathrm{C}$ in an ambient chamber. Escherichia coli strain ATCC 25922 was used for quality control. As shown in Table 1, the two strains isolated from calf A and calf B showed resistance to penicillin G, ampicillin, cephalexin, amoxicillin, lincomycin, roxithromycin, tetracycline, doxycycline, rifampin, azithromycin, metronidazole, clarithromycin, and vancomycin, but they were sensitive to ciprofloxacin, enrofloxacin, norfloxacin, levofloxacin, lomefloxacin, fosfomycin, nitrofurantion, florfenicol, trimethoprim sulfamethoxazole and trimethoprim.

\section{Discussion and conclusions}

While there have been few reports of E. hormaechei associated with infection in animals, only a single uterine infection in a fox and intestinal infection in a piglet have been reported [5, 6]. E. hormaechei can be isolated from sputum, urine, exudate, wounds, blood, tissue, and organs in patients $[1,4,9-15]$. E. hormaechei infection is common in patients in intensive care and in infants, who often contract it through contaminated nutrition 
Table 1 The results of drug sensitive test

\begin{tabular}{|c|c|c|c|c|c|c|c|}
\hline \multirow[t]{2}{*}{ Name } & \multirow[t]{2}{*}{ Sensitivity } & \multicolumn{2}{|c|}{ Diameter } & \multirow[t]{2}{*}{ Name } & \multirow[t]{2}{*}{ Sensitivity } & \multicolumn{2}{|c|}{ Diameter } \\
\hline & & HN447 & HN449 & & & HN447 & HN449 \\
\hline Penicillin G & $R$ & 0 & 0 & Tetracycline & $\mathrm{R}$ & 0 & 0 \\
\hline Ampicillin & $\mathrm{R}$ & 0 & 0 & Doxycycline & $\mathrm{R}$ & 0 & 0 \\
\hline Cephalexin & $\mathrm{R}$ & 0 & 0 & Minocycline & $S / I$ & 18 & 15 \\
\hline Amoxicillin & $\mathrm{R}$ & 0 & 0 & Fosfomycin & $S$ & 17 & 20 \\
\hline Ciprofloxacin & S & 25 & 24 & Nitrofurantion & S & 20 & 22 \\
\hline Enrofloxacin & S & 26 & 30 & Rifampin & $\mathrm{R}$ & 0 & 0 \\
\hline Norfloxacin & S & 23 & 30 & Azithromycin & $\mathrm{R}$ & 0 & 0 \\
\hline Levofloxacin & S & 32 & 28 & Metronidazole & $\mathrm{R}$ & 0 & 0 \\
\hline Lomefloxacin & S & 28 & 30 & Clarithromycin & $\mathrm{R}$ & 0 & 0 \\
\hline Lincomycin & $\mathrm{R}$ & 0 & 0 & Florfenicol & $S$ & 25 & 25 \\
\hline Kanamycin & I/S & 17 & 20 & Trimethoprim sulfamethoxazole & S & 24 & 28 \\
\hline Gentamicin & 1 & 14 & 14 & Trimethoprim & $S$ & 23 & 27 \\
\hline Roxithromycin & $\mathrm{R}$ & 0 & 0 & Vancomycin & $\mathrm{R}$ & 0 & 0 \\
\hline
\end{tabular}

$R$ Resistant, I Intermediate sensitivity, $S$ Sensitive, Diameter Diameter of inhibitive zone $(\mathrm{mm})$. The zone diameter (mm) interpretive criteria for drugs: Penicillin $\mathrm{G}$, Ampicillin and Amoxicillin: $S \geq 17, \mathrm{l}: 14-16, R \leq 13$; Ciprofloxacin: $S \geq 15, R \leq 14$; Enrofloxacin and Lomefloxacin: $S \geq 22, I: 19-21, R \leq 18$; Norfloxacin: $S \geq 17, I: 13-16$, $R \leq 12$; Levofloxacin: $S \geq 17, \mathrm{I}: 14-16, R \leq 13$; Lincomycin: $S \geq 13, R \leq 12$; Kanamycin: $S \geq 18, I: 14-17, R \leq 13$; Gentamicin: $S \geq 15, I: 13-14, R \leq 12 ;$ Roxithromycin: $S \geq 13$, $R \leq 12$; Tetracycline: $S \geq 15$, I:12-14, $R \leq 11$; Doxycycline: $S \geq 14, \mathrm{I}: 11-13, \mathrm{R} \leq 10$; Minocycline: $\mathrm{S} \geq 16, \mathrm{I}: 13-15, \mathrm{R} \leq 12 ;$ Fosfomycin: $\mathrm{S} \geq 16, \mathrm{l}: 13-15, \mathrm{R} \leq 12$; Nitrofurantion: $S \geq 17, \mathrm{I}: 15-16, R \leq 14$; Rifampin: $S \geq 20$, I:17-19, $R \leq 16$; Azithromycin: $S \geq 21, \mathrm{l}: 18-20, R \leq 17$; Metronidazole: $S \geq 17, \mathrm{l}: 14-16, \mathrm{R} \leq 13$; Clarithromycin: $S \geq 21$, I:18-20, $R \leq 17$; Florfenicol: $S \geq 22$, I:19-21, $R \leq 18$; Trimethoprim sulfamethoxazole: $S \geq 16$, I:11-15, $R \leq 10$; Trimethoprim: $S \geq 16$, I:11-15, $R \leq 10$; Vancomycin: $S \geq 16,1: 4-8, R \leq 2$

$[16,17]$; these findings indicate the immunological incompetence host would be susceptible to E. hormaechei. In this case, two 2-month-old calves showed that respiratory disease was associated with E. hormaechei; this was confirmed by $16 \mathrm{~S}$ rRNA gene sequencing and biochemical tests, and these two calves were not weaned. Considering the infection routes found in infants [11, $17]$, the feeding pathway might be a means by which $E$. hormaechei spreads in calves. Previous studies have reported that $E$. hormaechei can contaminate infant formula, and such cases have been found in Italy, the Czech Republic, and Holland [11, 18], so the risk of animal-to-human transmission is not negligible.

A large number of respiratory diseases have been described in calves, with pathogens including a variety of bacteria [19], but no report of calf pneumonia associated with E. hormaechei has been published. Compared to the signs of the calf infected by other bacterium such as Pasteurella multocida, non-specific clinical findings were observed in this case, and this may lead to misdiagnosis and inappropriate treatment. Currently, $\beta$-lactam antimicrobials (penicillin G, ampicillin, cephalexin, and amoxicillin) are extensively used in the treatment of calf respiratory infections in China [20]. In this case, the first calf was treated with penicillin $\mathrm{G}$ for 5 days, but it still died. Drug sensitivity testing showed that the E. hormaechei strains isolated from the lungs of calf A were completely resistant to $\beta$-lactam antimicrobials, so the failure of treatment of the first calf was not unexpected; the strains were also completely resistant to the nine other antimicrobials. Similarly, the strain isolated from the dead fox showed the same resistance to $\beta$-lactam antimicrobials and tetracycline antibiotics and the same sensitivity to quinolones (ciprofloxacin and enrofloxacin) [5]. O'Hara reported that the majority of strains isolated from humans are resistant to ampicillin, cefoxitin, and cephalothin [1], and a strain isolated from a female inpatient in Brazil was also resistant to all of the $\beta$-lactam antimicrobials tested, and it harbored two mobile genetic elements (Tn4401 and ISAba125) which play roles in the production of Klebsiella pneumoniae carbapenemase and New Delhi metallo- $\beta$-lactamase, respectively [12]. Whether the isolates in this case share this mechanism of resistance to $\beta$-lactam drugs remains unclear. Lately, in China, the strains isolated from patients have shown that mobile genetic elements could transmit their ability to resist $\beta$-lactam to the wild-type recipient bacterium [15], and additional investigations are warranted to address this issue.

To the best of our knowledge, this is the first case report of calves showing respiratory disease to be associated with $E$. hormaechei. The presence of this pathogen in the two calves separated by an interval of 1 week in the same farm suggests that the E. hormaechei might be a pathogen in calves. However, the limited number of animals (2) involved in this case is not sufficient to allow us to draw that conclusion. Broader investigations of $E$. hormaechei associated with respiratory disease are need 
to establish the relationship between the disease and the bacteria. A future work evaluating calves experimentally infected with the isolated strains would help establish the pathogenicity of $E$. hormaechei in the new emerging host and whether the infection routes through feeding pathway found in infants $[11,17]$ was utilized by $E$. hormaechei in spreading in calves could be investigated in further research.

\section{Supplementary information}

Supplementary information accompanies this paper at https://doi.org/10. 1186/s12917-019-2207-z.

Additional file 1. Calf's clinical appearance. Abdominal respiration movements were observed.

\section{Abbreviations}

$16 S$ rRNA: 165 ribosomal RNA; E. hormaechei: Enterobacter hormaechei

\section{Acknowledgments}

We thank LetPub (www.letpub.com) for its linguistic assistance during the preparation of this manuscript.

\section{Authors' contributions}

ZW and LD participated in the sample collection, pathogen isolation, histopathological test, drug sensitive test and $16 \mathrm{~S}$ rRNA detection. FL, YH and $C L$ participated in the data analyzing. LY, YK and HS participated in the design of the study, drafted the main parts of the manuscript as well as revised the manuscript. All authors read and approved the final manuscript.

\section{Funding}

The National Natural Science Foundation of China (Grant no. 31371381) supported the design of the study and writing the manuscript, the Henan Provincial Scientific and technological research project (Grant nos. 182102110084 and 162102110046 ) supported the sample collection and analysis respectively, and the program for Innovative Research Team of Science and Technology in University of Henan Province (No. 20IRTSTHN024) and Nanyang Normal University (CN) (Grant no. 15081) supported interpretation of data in this study.

\section{Availability of data and materials}

All data generated or analyzed during this study are included in this published article Sequences obtained in the present study are deposited in GenBank under accession numbers MK774673 and MK774674.

\section{Ethics approval and consent to participate}

Consent for participation is not applicable to this study. The research protocol was approved by the Animal Welfare and Ethics Committee of Nanyang Normal University (approval number: 18032, year: 2018).

\section{Consent for publication}

The owners of the animals in the case report gave written consent to publish the case in public.

\section{Competing interests}

The authors declare that they have no competing interests.

Received: 19 April 2019 Accepted: 4 December 2019

Published online: 03 January 2020

\section{References}

1. O'Hara CM, Steigerwalt AG, Hill BC, Farmer JJ 3rd, Fanning GR, Brenner DJ. Enterobacter hormaechei, a new species of the family enterobacteriaceae formerly known as enteric group 75. J Clin Microbiol. 1989;27:2046-9.

2. Halda-Alija L, Hendricks SP, Johnston TC. Spatial and temporal variation of enterobacter genotypes in sediments and the underlying hyporheic zone of an agricultural stream. Microb Ecol. 2001;42:286-94.
3. Davin-Regli A, Bosi C, Charrel R, Ageron E, Papazian L, Grimont PA, Cremieux A, Bollet C. A nosocomial outbreak due to enterobacter cloacae strains with the e. Hormaechei genotype in patients treated with fluoroquinolones. J Clin Microbiol. 1997:35:1008-10.

4. Paauw A, Caspers MP, Leverstein-van Hall MA, Schuren FH, Montijn RC, Verhoef J, Fluit AC. Identification of resistance and virulence factors in an epidemic enterobacter hormaechei outbreak strain. Microbiology. 2009;155: 1478-88.

5. Shan-Shan W, Yun-Jia S, Xing-Yang C, Cheng-Wei W, Shan-Shan GU, Xin Y, Shuang $X$, Jun-Wei GE, Hong-Yan C. Isolation,identification and phylogenetic analysis of enterobacter hormaechei from foxes. Chin Vet Sci. 2017:47:768-72.

6. Lu-Yao LI, Liu MJ, Teng MM, Wang L, Zhang YX, Liu BQ. Study on the biological characteristics of enterobacter hormaechei. J Ani Sci Vet Med. 2017:36:1-6.

7. Jiang H, Dong H, Zhang G, Yu B, Chapman LR, Fields MW. Microbial diversity in water and sediment of lake chaka, an athalassohaline lake in northwestern China. Appl Environ Microbiol. 2006;72:3832-45.

8. CLSI: Performance standards for antimicrobial susceptibility testing, 29th edition: CLSI M100-S29; 2018

9. Carvalho-Assef AP, Pereira PS, Albano RM, Beriao GC, Tavares CP, Chagas TP, Marques EA, Timm LN, Da Silva RC, Falci DR, et al. Detection of ndm-1-, ctxm-15-, and qnrb4-producing enterobacter hormaechei isolates in Brazil. Antimicrob Agents Chemother. 2014;58:2475-6.

10. Daurel C, Fiant AL, Bremont S, Courvalin P, Leclercq R. Emergence of an enterobacter hormaechei strain with reduced susceptibility to tigecycline under tigecycline therapy. Antimicrob Agents Chemother. 2009;53:4953-4.

11. Giammanco GM, Aleo A, Guida I, Mammina C. Molecular epidemiological survey of citrobacter freundii misidentified as cronobacter spp. (enterobacter sakazakii) and enterobacter hormaechei isolated from powdered infant milk formula. Foodborne Pathog Dis. 2011;8:517-25.

12. Pereira PS, Borghi M, Albano RM, Lopes JC, Silveira MC, Marques EA, Oliveira JC, Asensi MD. Carvalho-Assef AP. Coproduction of ndm-1 and kpc-2 in enterobacter hormaechei from Brazil. Microb Drug Resist. 2015;21:234-6.

13. Rafferty B, Dolgilevich S, Kalachikov S, Morozova I, Ju J, Whittier S, Nowygrod R, Kozarov E. Cultivation of enterobacter hormaechei from human atherosclerotic tissue. J Atheroscler Thromb. 2011;18:72-81.

14. Sampaio JL, Ribeiro VB, Campos JC, Rozales FP, Magagnin CM, Falci DR, da Silva RC, Dalarosa MG, Luz DI, Vieira FJ, et al. Detection of oxa-370, an oxa48-related class d beta-lactamase, in enterobacter hormaechei from Brazil. Antimicrob Agents Chemother. 2014;58:3566-7.

15. Yang B, Feng Y, McNally A, Zong Z. Occurrence of enterobacter hormaechei carrying blandm-1 and blakpc-2 in China. Diagn Microbiol Infect Dis. 2018; 90:139-42.

16. Rottman M, Benzerara Y, Hanau-Bercot B, Bizet C, Philippon A, Arlet G. Chromosomal ampc genes in enterobacter species other than enterobacter cloacae, and ancestral association of the act-1 plasmid-encoded cephalosporinase to enterobacter asburiae. FEMS Microbiol Lett. 2002;210: 87-92.

17. Campos LC, Lobianco LF, Seki LM, Santos RM, Asensi MD. Outbreak of enterobacter hormaechei septicaemia in newborns caused by contaminated parenteral nutrition in Brazil. J Hosp Infect. 2007;66:95-7.

18. Townsend SM, Hurrell E, Caubilla-Barron J, Loc-Carrillo C, Forsythe SJ. Characterization of an extended-spectrum beta-lactamase enterobacter hormaechei nosocomial outbreak, and other enterobacter hormaechei misidentified as cronobacter (enterobacter) sakazakii. Microbiology. 2008; 154:3659-67.

19. Griffin D, Chengappa MM, Kuszak J, McVey DS. Bacterial pathogens of the bovine respiratory disease complex. Vet Clin North Am Food Anim Pract. 2010;26:381-94

20. Han ML, Kang LC, Zhong FG, Huang $X$, Tan PF. Research progress on bacterial pathogens of the bovine respiratory disease. Chi Ani Hus Vet Med. 2013:40:165-72

\section{Publisher's Note}

Springer Nature remains neutral with regard to jurisdictional claims in published maps and institutional affiliations. 